\title{
FEFFERMAN-GRAHAM AMBIENT METRICS OF PATTERSON-WALKER METRICS
}

\author{
MATTHIAS HAMMERL, KATJA SAGERSCHNIG, JOSEF ŠILHAN, \\ ARMAN TAGHAVI-CHABERT, AND VOJTĚCH ŽÁDNÍK
}

\begin{abstract}
Given an $n$-dimensional manifold $N$ with an affine connection $D$, we show that the associated Patterson-Walker metric $g$ on $T^{*} N$ admits a global and explicit Fefferman-Graham ambient metric. This provides a new and large class of conformal structures which are generically not conformally Einstein but for which the ambient metric exists to all orders and can be realized in a natural and explicit way. In particular, it follows that Patterson-Walker metrics have vanishing FeffermanGraham obstruction tensors. As an application of the concrete ambient metric realization we show in addition that Patterson-Walker metrics have vanishing Q-curvature.
\end{abstract}

\section{INTRODUCTION AND MAIN RESULT}

Given a signature $(p, q)$ conformal structure $[g]$ on an $m=p+q$ dimensional manifold $M$, it was shown in seminal work by Charles Fefferman and C. Robin Graham (see FG85, FG12]) that under specific conditions the conformal structure can be encoded equivalently as a signature $(p+1, q+1)$ pseudo-Riemannian metric ( $\mathbf{M}, \mathbf{g})$ with vanishing Ricci curvature. This description has been fundamental in constructing and classifying conformal invariants (see e.g. FG85, BEG94]) and for constructing and studying conformally invariant differential operators (see Gra92, GJMS92).

To build the Fefferman-Graham ambient metric for given local coordinates $x$ on $M$, one first considers the ray bundle of metrics in the conformal class $[g]$, written as $\mathbb{R}_{+} \times \mathbb{R}^{m}$ with coordinates $(t, x)$. The ambient space $\mathbf{M}$ is obtained by adding a new transversal coordinate $\rho \in \mathbb{R}$, and then an ansatz for the Fefferman-Graham ambient metric $\mathbf{g}$ is

$$
\mathbf{g}=t^{2} g_{i j}(x, \rho) d x^{i} \odot d x^{j}+2 \rho d t \odot d t+2 t d t \odot d \rho,
$$

where $g=g_{i j}(x, 0) d x^{i} d x^{j}$ is a representative metric in the conformal class. It is directly visible from the formula that $\mathbf{g}$ is homogeneous of degree 2 with respect to the Euler field $t \partial_{t}$ on $\mathbf{M}$.

To show existence of a Fefferman-Graham ambient metric $\mathbf{g}$ for given $g$, the ansatz (1) determines an iterative procedure to determine $g_{i j}(x, \rho)$ as a Taylor series in $\rho$ satisfying $\operatorname{Ric}(\mathbf{g})=0$ to infinite order at $\rho=0$. For $m$ odd existence (and a natural version of uniqueness) of $\mathbf{g}$ as an infinityorder series expansion in $\rho$ is guaranteed for general $g_{i j}(x)$. For $m=2 n$

Date: October 19, 2018.

2000 Mathematics Subject Classification. 53A30, 53A55, 53B30.

Key words and phrases. Fefferman-Graham ambient metrics, Patterson-Walker metrics, Conformal geometry, Q-curvature.

KS is an INdAM (Istituto Nazionale di Alta Matematica) research fellow.

JŠ was supported by the Czech science foundation (GAČR) under grant P201/12/G028. 
even, the existence of an infinity order jet for $g_{i j}(x, \rho)$ with $\operatorname{Ric}(\mathbf{g})=0$ asymptotically at $\rho=0$ is obstructed at order $n$. Existence of $g_{i j}(x, \rho)$ as an infinity order series expansion in $\rho$ with $\operatorname{Ric}(\mathbf{g})=0$ asymptotically at $\rho=0$ is then equivalent to vanishing of the Fefferman-Graham obstruction tensor $\mathcal{O}$, which is a conformal invariant. Existence of $\mathbf{g}$ for $m=2 n$ even does not in general guarantee uniqueness.

Results which provide global Fefferman-Graham ambient metrics, where g can then be constructed in a natural way from $g$ and satisfies $\operatorname{Ric}(\mathbf{g})$ globally and not just asymptotically at $\rho=0$ are rare, both in the odd- and even-dimensional situation. A special instance where global ambient metrics can at least be shown to exist occurs for $g$ real-analytic, and $m$ either being odd or $m$ even and with obstruction tensor $\mathcal{O}$ of $g$ vanishing. The simplest case of geometric origin for which one has global ambient metrics consists of locally conformally flat structures $(M,[g])$, where $(\mathbf{M}, \mathbf{g})$ exists and is unique up to diffeomorphisms (see FG12, ch. 7). Another well known geometric case are conformal structures $(M,[g])$ which contain an Einstein metric $g$ : If $\operatorname{Ric}(g)=2 \lambda(m-1) g$, then $\mathbf{g}$ on $\mathbb{R}_{+} \times M \times \mathbb{R}$ can be written directly in terms of $g$ as

$$
\mathbf{g}=t^{2}(1+\lambda \rho)^{2} g+2 \rho d t \odot d t+2 t d t \odot d \rho .
$$

In work by Thomas Leistner and Pawel Nurowski it was shown that the so called $p p$-waves admit global ambient metrics in the odd-dimensional case and under specific assumptions in the even-dimensional case, see [LN10]. Concrete and explicit ambient metrics for specific examples of families of conformal structures induced by generic 2-distributions on 5-manifolds and generic 3-distributions on 6 manifolds have been constructed in Nur08, LN12, Wil14, ALN15.

The present article expands the class of metrics for which canonical ambient metrics exist globally to Patterson-Walker metrics: Given an affine connection $D$ on an $n$-manifold $N$ with $n \geq 2$, which is supposed to be torsion-free and to preserve a volume form, the Patterson-Walker metric $g$ is a natural split-signature $(n, n)$ metric on $T^{*} N$, see recent work HSSTZ16] for historical background on Patterson-Walker metrics, references and a modern treatment. Our main result is:

Theorem 1. Let $D$ be a torsion-free affine connection on $N$ which preserves a volume form. Denote local coordinates on $N$ by $x^{A}$ and the induced canonical fibre coordinates on $T^{*} N$ by $p_{A}$. Let $\Gamma_{A}{ }_{B}{ }_{B}$ denote the Christoffel symbols of D. Let

$$
g=2 \mathrm{~d} x^{A} \odot \mathrm{d} p_{A}-2 \Gamma_{A}{ }_{B}{ }_{B} p_{C} \mathrm{~d} x^{A} \odot \mathrm{d} x^{B}
$$

be the Patterson-Walker metric induced on $T^{*} N$ by $D$. Then, with $\operatorname{Ric}_{A B}$ the Ricci curvature of $D$,

$$
\begin{aligned}
& \mathbf{g}=2 \rho d t \odot d t+2 t d t \odot d \rho \\
& +t^{2}\left(2 d x^{A} \odot d p_{A}-2 p_{C} \Gamma_{A}{ }^{C}{ }_{B} d x^{A} \odot d x^{B}+\frac{2 \rho}{n-1} \operatorname{Ric}_{A B} d x^{A} \odot d x^{B}\right)
\end{aligned}
$$

is a globally Ricci-flat Fefferman-Graham ambient metric for the conformal class $[g]$. 
We note that as an immediate consequence of the existence of the ambient metric for $(M,[g])$, the conformally invariant Fefferman-Graham obstruction tensor $\mathcal{O}$ associated to $[g]$ vanishes.

It is not difficult to check Ricci-flatness of (4) directly: Specifically, one employs formula (3.17) of [FG85], which is applicable to any ambient metric in normal form (11). The computation is then based on the following key facts: The Ricci curvature of the Patterson-Walker metric $g$ is up to a constant multiple just the pullback of the Ricci curvature of $D$ and this tensor and its covariant derivative are totally isotropic, see [HSSTZ16]. We note that formula (4) for $\mathbf{g}$ says that the ambient metric is in fact linear in $\rho$ and the iterative procedure determining the ambient metric stops after the first step.

A geometric proof of vanishing Ricci curvature is presented in the next section. This is based on a combination of the well known Patterson-Walker and Thomas cone constructions, both of which we recall.

\section{Geometric construction of the ambient metric}

The association $D \rightsquigarrow g$ generalizes to a natural association from projective to conformal structures. Recall that two affine connections $D, D^{\prime}$ on $N$ are called projectively related or equivalent if they have the same geodesics as unparameterized curves, which is the case if and only if there exists a 1-form $\Upsilon \in \Omega^{1}(N)$ with

$$
D_{X}^{\prime} Y=D_{X} Y+\Upsilon(X) Y+\Upsilon(Y) X
$$

for all $X, Y \in \mathfrak{X}(N)$. It is sufficient to restrict ourselves to special connections in a projective class, i.e., to those that preserve some volume form. For projective structures it is useful to employ a suitably scaled projective density bundle $\mathcal{E}_{+}(1)$, defined as the special case of weight $w=1$ of $\mathcal{E}(w):=\left(\wedge^{n} T N\right)^{-\frac{w}{n+1}}$. Then a section $s: N \rightarrow \mathcal{E}_{+}(1)$ corresponds to a choice of a special affine connection $D$ in the projective equivalence class $[D]$, and any $s^{\prime}=e^{f} s$ corresponds to $D^{\prime}$ projectively related to $D$ via (5) with $\Upsilon=d f$. We define $M=T^{*} N(2)$ the (projectively) weighted co-tangent bundle of $N$. Then, as was shown in [HSSTZ16, two projectively related affine connections $D, D^{\prime}$ on $N$ induce two conformally related metrics $g, g^{\prime}$ on $M$, and we therefore have a natural association $(N,[D]) \rightsquigarrow(M,[g])$.

The cone $\mathcal{C}:=\mathcal{E}_{+}(1)$ carries the canonical and well known Ricci-flat Thomas cone connection $\nabla$, see [Tho34] or [ČS09], where the specific weight 1 (different from [ČS09]) is most convenient for our computations. We will need a local formula for $\nabla$ : Let $s: N \rightarrow \mathcal{E}_{+}(1)$ be the scale corresponding to an affine connection $D \in[D]$, providing a trivialization $\mathcal{E}_{+}(1) \cong \mathbb{R}_{+} \times N$ via $\left(x^{0}, x\right) \mapsto s(x) x^{0}$. In this trivialization the Thomas cone connection is given by

$$
\nabla_{X} Y=D_{X} Y-\frac{1}{n-1} \operatorname{Ric}(X, Y) Z, \nabla Z=\operatorname{id}_{T \mathcal{C}}
$$

where $X, Y \in \mathfrak{X}(N)$ and $Z=x^{0} \partial_{x^{0}}$ is the Euler field on $\mathcal{C}$. It is in fact easy to see directly from formula (6) that the thus defined affine connection $\nabla$ on the Thomas cone $\mathcal{C}$ is independent of the choice of scale and Ricci-flat. 
Employing a local coordinate patch on $N$ which induces coordinates $x^{A}, y_{A}$ on the co-tangent bundle $T^{*} N$ and coordinates $x^{0}, x^{A}, y_{A}, y_{0}$ on $T^{*} \mathcal{C} \cong \mathbb{R}_{+} \times T^{*} N \times \mathbb{R}$, the Patterson-Walker metric $\mathbf{g}$ associated to $\nabla$ is

$$
\begin{aligned}
\mathbf{g}= & 2 d x^{A} \odot d y_{A}+2 d x^{0} \odot d y_{0}-\frac{4}{x^{0}} y_{B} d x^{0} \odot d x^{B} \\
& -2 y_{C} \Gamma_{A}{ }^{C}{ }_{B} d x^{A} \odot d x^{B}+2 \frac{x^{0} y_{0}}{n-1} \operatorname{Ric}_{A B} d x^{A} \odot d x^{B} .
\end{aligned}
$$

Ricci-flatness of $\mathbf{g}$ follows directly from Ricci-flatness of $\nabla$, see Theorem 2 of HSSTZ16]. Via the change of coordinates $t=x^{0}, \rho=\frac{y_{0}}{x^{0}}, p_{A}=\frac{y_{A}}{\left(x^{0}\right)^{2}}$ the metric $\mathrm{g}$ transforms to (4), which is the form of a Fefferman-Graham ambient metric (11). In particular this shows Theorem 1.

Remark 2.1. As a Patterson-Walker metric $(\mathbf{M}, \mathbf{g})$ carries a naturally induced homothety $\mathbf{k}$ of degree 2 , which takes the form $2 p_{A} \partial_{p_{A}}+2 \rho \partial_{\rho}$. According to Lemma 5.1 of [HSSTZ16] the infinitesimal affine symmetry $Z$ of $\nabla$ lifts to a Killing field, which one computes as $t \partial_{t}-2 p_{A} \partial_{p_{A}}-2 \rho \partial_{\rho}$. In particular it follows that the Euler field $t \partial_{t}$ of the Fefferman-Graham ambient metric $\mathbf{g}$ can be written as the sum of this Killing field and the homothety $\mathbf{k}$. $T \mathbf{M}$ carries the maximally isotropic $(n+1)$-dimensional subspace spanned by $\left\{\partial_{p_{A}}, \partial_{\rho}\right\}$ which is preserved by $\nabla$. This subspace can be equivalently described by a $\boldsymbol{\nabla}$-parallel pure spinor $\mathbf{s}$ on $\mathbf{M}$. The ambient Killing field $\mathbf{k}$ and the ambient parallel pure spinor $\mathbf{s}$ correspond to a homothety $k$ of $g$ and a parallel pure spinor $\chi$ on $M$ that belong to the characterizing objects of the Patterson-Walker metric $g$, see Theorem 1 of [HSSTZ16].

We conclude this section by summarizing the construction:

Theorem 2. Given a projective structure $(N,[D])$ on an $n$-dimensional manifold $N$, the geometric constructions indicated in the following diagram commute:

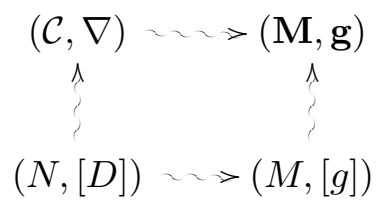

In particular, the induced conformal structure $[g]$ admits a globally Ricciflat Fefferman-Graham ambient metric $\mathbf{g}$ which is itself a Patterson-Walker metric.

We remark here that, for generic $[D]$, the resulting conformal class $[g]$ does not contain an Einstein metric, see Theorem 2 of [HSSTZ16. In particular, one obtains a large class of conformal structures which are not conformally Einstein but which admit globally Ricci-flat and explicit ambient metrics.

\section{VAnishing $Q-$ CURVATURE}

The $Q$-curvature $Q_{g}$ of a given metric $g$ is a Riemannian scalar invariant with a particularly simple transformation law with respect to conformal change of metric. It has been introduced by T. Branson in Bra93] and has been the subject of intense research in recent years, see e.g. [CEOY08. for an overview. Computation of $Q$-curvature is notoriously difficult, see e.g. 
GP03. An explicit form of a Fefferman-Graham ambient metric $\mathbf{g}$ for a given metric $g$ allows a computation of $Q_{g}$. Using the fact that $\mathbf{g}$ is actually a Patterson-Walker metric, this computation is particularly simple.

Theorem 3. The Patterson-Walker metric g associated to a volume-preserving, torsion-free affine connection $D$ has vanishing $Q$-curvature $Q_{g}$.

Proof. We follow the computation method for $Q_{g}$ from [FH03]: For this it is necessary to compute $-\boldsymbol{\Delta}^{n} \log (t)$, where $\boldsymbol{\Delta}$ is the ambient Laplacian on $\mathbf{M}=\mathbb{R}_{+} \times T^{*} N \times \mathbb{R}$ associated to $\mathbf{g}$ and $t: \mathbf{M} \rightarrow \mathbb{R}_{+}$is the first coordinate. Restricting $-\boldsymbol{\Delta}^{n} \log (t)$ to the cone $\mathbb{R}_{+} \times T^{*} N \times\{0\}$ and evaluating at $t=$ 1 yields $Q_{g}$ (see [FH03]). To show that Q-curvature vanishes for $g$, it is in particular sufficient to show that $\Delta \log (t)=0$. However, the function $t: \mathbf{M} \rightarrow \mathbb{R}_{+}$is horizontal since it is just the pullback of the coordinate function $x^{0}: \mathcal{E}_{+}(1) \rightarrow \mathbb{R}_{+}$on the Thomas cone $\mathcal{C} \cong \mathbb{R}_{+} \times N$ via the canonical projection $T^{*} \mathcal{C} \rightarrow \mathcal{C}$. It follows from the explicit formula for the Christoffel symbols of a Patterson-Walker metric that $\boldsymbol{\Delta}$ vanishes on any horizontal function, see [HSSTZ16], section 2.1. Thus in particular $\boldsymbol{\Delta} \log (t)=0$, and then also $Q_{g}=0$.

\section{REFERENCES}

[BEG94] T.N. Bailey, M. Eastwood, and C.R. Graham. Invariant theory for conformal and CR geometry. Ann. of Math. (2), 139(3):491-552, 1994.

[Bra93] T. P. Branson. The functional determinant, volume 4 of Lecture Notes Series. Seoul National University, Research Institute of Mathematics, Global Analysis Research Center, Seoul, 1993.

[CEOY08] S.-Y. A. Chang, M. Eastwood, B. Orsted, and P. C. Yang. What is Qcurvature? Acta Appl. Math., 102(2-3):119-125, 2008.

[ČS09] A. Čap and J. Slovák. Parabolic Geometries I: Background and General Theory. Mathematical Surveys and Monographs. American Mathematical Society, Providence, RI, 2009.

[FG85] C. Fefferman and C.R. Graham. Conformal invariants. Astérisque, (Numero Hors Serie):95-116, 1985. The mathematical heritage of Élie Cartan (Lyon, 1984).

[FG12] C. Fefferman and C. R. Graham. The Ambient Metric. Annals of Mathematics Studies 178. Princeton, NJ: Princeton University Press. v, 2012.

[FH03] C. Fefferman and K. Hirachi. Ambient metric construction of $Q$-curvature in conformal and CR geometries. Math. Res. Lett., 10(5-6):819-831, 2003.

[GJMS92] C.R. Graham, R. Jenne, L.J. Mason, and G.A.J. Sparling. Conformally invariant powers of the Laplacian. I. Existence. J. London Math. Soc. (2), 46(3):557$565,1992$.

[GP03] A.R. Gover and L.J. Peterson. Conformally invariant powers of the Laplacian, Q-curvature, and tractor calculus. Comm. Math. Phys., 235(2):339-378, 2003.

[Gra92] C.R. Graham. Conformally invariant powers of the Laplacian. II. Nonexistence. J. London Math. Soc. (2), 46(3):566-576, 1992.

[HSSTZ16] M. Hammerl, K. Sagerschnig, J. Šilhan, A. Taghavi-Chabert, and V. Žádník. Conformal Patterson-Walker metrics. arXiv:1604.08471.

[ALN15] I. M. Anderson, T. Leistner and P. Nurowski. Explicit ambient metrics and holonomy. arXiv:1501.00852, 2015.

[LN10] T. Leistner and P. Nurowski. Ambient metrics for $n$-dimensional $p p$-waves. Comm. Math. Phys., 296(3):881-898, 2010.

[LN12] T. Leistner and P. Nurowski. Ambient metrics with exceptional holonomy. Ann. Sc. Norm. Super. Pisa Cl. Sci. (5), 11(2):407-436, 2012.

[Nur08] P. Nurowski. Conformal structures with explicit ambient metrics and conformal $G_{2}$ holonomy. In Symmetries and overdetermined systems of partial differential 
equations, volume 144 of IMA Vol. Math. Appl., pages 515-526. Springer, New York, 2008

[Tho34] T.Y. Thomas. The Differential Invariants of Generalized Spaces. 1934.

[Wil14] T. Willse. A missing distribution and an explicit ambient metric of holonomy $g_{2}^{*}$. 2014. arXiv:1411.7172.

M. H.: University of Greifswald, Department of Mathematics and Informatics, Walther-Rathenau-Str. 47, 17489 Greifswald, Germany

K. S.: Indam-Politecnico di Torino, Dipartimento di Scienze Matematiche, CORso DuCA DEgli Abruzzi 24, 10129 Torino, ItAly

J. Š. And A. T.-C..: Masaryk University, Faculty of Science, KotláŘská 2, 61137 Brno, Czech Republic

V. Ž: Masaryk University, Faculty of Education, Poříčí 31, 60300 Brno, Czech REPUBLIC

E-mail address: matthias.hammerl@univie.ac.at, katja.sagerschnig@univie.ac.at, silhan@math.muni.cz, taghavia@math.muni.cz, zadnik@mail.muni.cz 